\title{
The role of context in episodic memory
}

\author{
WILLIAM BEVAN and JAMES N. FEUER \\ Duke University, Durham, North Carolina 27706
}

\begin{abstract}
Subjects were shown black and white drawings of common objects in a common, and therefore plausible, setting, in an unusual but plausible setting, or in a bizarre, that is, unusual and implausible, setting. The objects were also displayed on a homogeneous white background, that is, without a specific scenic context. Tests of both immediate and delayed free recall were made. Recall was best when the object was presented on the homogeneous white background. Among the three conditions involving a scenic context, performance was better when the settings were plausible and poorer when the setting was bizarre.
\end{abstract}

In a 1969 paper, Bevan and Avant examined the role of cortext in semantic memory. Their focal stimuli were colored pictures of common objects presented on a homogeneous white background and their contextual stimuli were colored pictures symmetrically arranged about each focal stimulus. The subjects' task was the free recall of the class names of the focal stimuli and the dependent variable was the thematic relationship between contextual and focal stimuli. Under one condition the contextual stimuli were members of the same conceptual class as the focal stimulus (e.g., if the focal stimulus was a dog, the contextual stimuli were also dogs); under a second, the contextual stimuli were members of a broader class that included the focal stimulus (e.g., if the focal stimulus was a dog, the contextual stimuli were other mammals: a goat, a horse, a cat, and a monkey); finally, under a third condition the contextual stimuli were a random selection of objects (e.g., a dog surrounded by a rolling pin, a flower, a chair, a bird). Contrary to expectation, the presence of contextual stimuli failed to facilitate recall; indeed, with contexts of intermediate and low relevance, the result appears to have been one of conceptual noise and, consequently, poorer performance than when the focal stimuli were presented alone. In a second experiment with class names rather than pictures presented as context, there was no reliable influence of context upon the free recall of the focal stimuli. However, in a third experiment, where the task was one of associative recall and the experimental strategy involved presentation of test displays consisting of the contextual stimuli only and with the subject requested to name the missing focal stimulus, goodness of recall proved to be a direct function of the degree of thematic relationship between focal and contextual stimuli.

In a subsequent study Bevan and Collyer (1969) employed the paradigm of the paired-associates experiment, with class names of common objects as stimulus

This experiment was conducted in the spring of 1975 as part of a larger independent study program carried out by J.N.F. under the supervision of W. B. words and CVCs as responses, or vice versa. In each case the contextual stimulus was a word in juxtaposition to the focal stimulus item: either the name of a member of the same class, of a broader category, or of a class unrelated to the word member of the stimulus pair. Thus, for example, if the word in a particular S-R pair was lion, the context words were, respectively: (1) tiger, panther, cougar, or leopard; (2) elephant, zebra, giraffe, or gorilla; (3) hammer, mimeograph, buckle, or clock. Here, again, the presence of a context word along with a focal stimulus word resulted in a decrement in performance, although the decrement when the thematic relation was high was only about half of that when it was low. In contrast, the presence of a context word with a response word facilitated performance, its influence being directly proportional to the specificity of the relationship between the two. Under the first condition the contextual stimulus was interpreted to have served as a distractor, and under the second as a mediating cue. This is in contrast to Postman and Phillips' (1964) earlier interpretation of the role of context words in terms of competing associations.

The present experiment deals with the role of context in episodic memory. The research strategy was to present the subject a series of scenes (black and white drawings) in which an easily recognized object (the focal stimulus) appeared in one of three kinds of settings: an appropriate setting, that is, a setting in which the stimulus object is likely to be found (e.g., a polar bear on a field of ice and snow with an igloo in the background); an unusual setting, that is, a setting in which it is possible but also highly unlikely that the stimulus object will be found (e.g., a polar bear on a sandy tropical beach with palm trees and a thatched hut in the background); and a bizarre setting, that is, a setting in which it is virtually impossible for the stimulus object to be found or a setting that defies simple reason (e.g., a polar bear balanced on the point of a tall church steeple). The experimental strategy, thus, simply involved showing the subjects a series of drawings representing the focal stimuli in one of the three settings or these same stimuli on a homogeneous white background, and then 
later requesting that they write down the names of as many of the objects as they could recall.

\section{METHOD}

\section{Design}

The experimental design was a mixed design with two between- and one within-subject variables. The between-subject variables were the (1) nature of the setting (context) and (2) time of free recall $(5 \mathrm{~min}$ or 5 days after presentation of the stimuli). The within-subject variable was stimulus objects.

\section{Subjects}

The subjects consisted of eight randomly organized groups of 12 undergraduate students each. Four groups, one for each of the types of stimulus display described above, were tested 5 min after presentation of the pictorial material. The remaining four were tested 5 days later.

\section{Stimulus Materials and Procedure}

The focal stimuli were black and white drawings of 16 easily recognized objects, special attention being given to planning the drawing so that the identity of the focal stimulus as well as its relation to its several settings would be clear. The 16 focal stimuli were a bugle, a book, a shoe, eyeglasses, a bicycle, a cowboy, a flag, a polar bear, a watch, an umbrella, a lamp, a chair, a gun, a car, a candelabra, and a tree. The three settings for one, the polar bear, have been described above. Other examples are as follows. Cowboy: The cowboy riding a horse; the cowboy riding a horse backward; the cowboy straddling a crescent moon. Lamp: The lamp on an end table; the lamp underneath an end table; the lamp on top of a jet in flight. Watch: The watch on someone's wrist; the watch around someone's ankle; the watch around a tree trunk.

Subjects, tested in groups, were shown each stimulus display once, projected for $5 \mathrm{sec}$ on a screen before them. Their prior instructions were that a series of drawings of common objects was to be presented serially on the screen. They were given no further information as to the purpose of the experiment although, when they were recruited, they had been told that they were to participate in a perception experiment. Five minutes or 5 days later they were reminded that they had been shown a series of pictures and were asked to write down the names of as many of the common objects as they could remember. Time allotted for recall in each instance was $3 \mathrm{~min}$.

\section{RESULTS AND DISCUSSION}

\section{The Main Effects}

An analysis of variance revealed all three independent variables to be significant sources of variance (between test periods: $\mathrm{F}=71.82, \mathrm{df}=1 / 88, \mathrm{p}<.001$; between stimuli: $\mathrm{F}=6.39, \mathrm{df}=15 / 1320, \mathrm{p}<.001$; between settings: $F=11.91$, df $=3 / 88, p<.001)$. Two of these effects are obviously to be expected and represent nothing of immediate special interest: Free recall after a 5 -day delay in testing was, on the average, $43 \%$ poorer than recall after a delay of $5 \mathrm{~min}$. Certain of the stimuli-for example, the book, bicycle, tree, and car-were consistently better recalled than others-for example, the candelabra, umbrella, lamp, and bugle. At the same time, it is worth noting that not a single interaction, first-order or second-order, proved to be significant. Thus, for example, the 5-min and 5-day performance levels, plotted from the four different conditions of the experiment, prove to be essentially parallel trends although derived from eight independent groups of subjects.

\section{Setting vs. No Setting in Recall of the Pictured Objects}

Figure 1 makes clear the general nature of the significant between-settings source of variance. Average performance appears to be better when no setting is presented with the focal stimulus than when one is present, regardless of its nature. Thus, the presence of context appears to interfere with recall. These results are similar to those of Bevan and Collyer (1969), Pan (1962), and Postman and Phillips (1964) when contexts were words presented along with focal-stimulus words in paired-associate recall and of Bevan and Avant (1969) when the stimuli, both focal and contextual, were pictures. However, the fact that interference was apparently less when the contexts were logically or plausibly related to the focal stimuli than when they were implausible or virtually impossible raises doubt about Postman and Phillips' explanation of the interference effect in terms of the competition of the focal and contextual S-R associations.

Several alternative explanations are possible. Considered in purely stimulus terms, the difference between the displays having no setting and those involving settings is one of physical complexity. Considered from the perspective of the subject, this could mean that the presence of the setting prompted search behavior, with attention divided between stimulus and background. This would be consistent with Bevan and

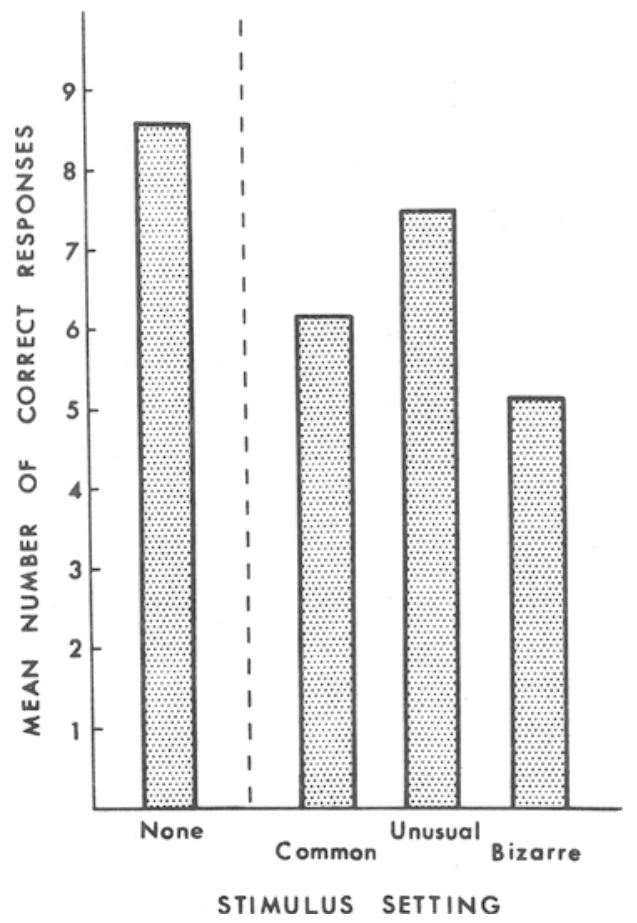

Figure 1. Free recall of drawings of common objects as a function of the type of context in which the focal stimulus appeared. Scores are the average of immediate and delayed recall tests. 
Collyer's (1969) suggestion that the context constitutes a distraction, with the plausible settings causing less distraction than the implausible. This possibility could be tested by having the subjects during the test identify not only the focal stimulus but the incident in which it figured as well. Hock (Note 1) reports that when he had subjects describe incidents during initial stimulus presentation, differences between plausible and implausible contexts disappeared. Related to the notion of distraction is the possibility that, with more complex displays, subjects were unclear as to what they should look at and thus failed to identify the focal stimulus (is the focal stimulus the cowboy or the horse?). However, care was taken to prepare the displays so that the focal stimulus was the prominent element, and interrogation following the test suggests that subjects had no trouble identifying the focal stimulus. Another possibility is that subjects, given the more complex displays, lacked sufficient time to encode the essential information for transfer to long-term storage. But the 5-sec exposure time of the present experiment exceeds the time usually reported to be necessary for information to pass through short-term memory, and Biederman (1972) has already demonstrated that essential information in complex photographs can be processed in periods as short as 300-700 msec.

If one reconstructed the events involved in the present task, they would most likely be as follows. On initial presentation the subject at first is aware of the presence of the display and only later recognizes its nature. Furthermore, the more complex the display, the longer the recognition time. Thus, with a fixed time for recognition, the more complex the display, the less likely the focal stimulus will be differentiated from its background. With free recall on demand, the subject most probably first constructs an image of the incident and then evaluates its content before reporting. Again, the more complex the display, the longer the evaluation time; conversely, with fixed periods for recall (an average of approximately $11 \mathrm{sec} /$ item), the more complex the display, the less likely the recall of the focal stimulus. Consistent with this is Kosslyn's (1975) recent observation that properties of small images take longer to evaluate than those of larger images, that only a limited amount of processing capacity is available for representing images, with the result that complex images are more degraded than simple, and that the construction of complex images takes longer than the construction of simple ones. Thus, when in recall attention is focused on one aspect of the image, display capacity is selectively allocated and evaluation must involve sequentially focusing on different aspects of the "image span." Should this be the case, then, when recall time is limited, the more complex the display, the less likely the recall of the focal stimulus.

\section{Free Recall as a Function of the Focal Stimulus-Context Relationship}

Comparison of recall for the several settings indicates performance to be better with plausible than with bizarre backgrounds [common and unusual vs bizarre: $t(70)=1.99, p<.05]$. Such results are consistent with the earlier reports of Collyer, Jonides, and Bevan (1972) and Wollen, Weber, and Lowry (1972) and contradict the long-standing belief of mnemonic experts that bizarre images are an important aid to recall. The fact that focal stimuli in unusual settings were recalled clearly better than when the settings were bizarre $[t(46)=2.84, p<.01]$ suggests that the value attributed to so-called bizarre images is a reflection of their novelty rather than their bizarreness. For an image to be maximally facilitative, the mnemonic rule should be revised to state that associations should be novel but at the same time plausible.

Why stimuli in unusual settings should be recalled better than the same stimuli in their most likely contexts is a problem that awaits empirical solution. Two possibilities have been proposed. Crowder (1976, pp. 116-117) suggests that when recall involves common, frequently experienced relationships, it need depend only on associated verbal processes. However, when relationships are uncommon, verbal processes are supplemented by the imagery system. Hock (Note 1), in contrast, associates the superiority of novel over familiar contexts with perceptual readiness. He believes that observers retain familiar scenes less well because they do not inspect them as carefully.

\section{REFERENCE NOTE்}

1. Hock, H. Personal communication. December 2. 1976.

\section{REFERENCES}

Bevan, W., \& Avant, L. L. The role of the thematic relationship between focal and contextual stimuli in recall. Journal of Verbal Learning and Verbal Behavior, 1969. 8. $185-190$.

Bevan, W., \& Collyer, S. C. The role of thematic relevance of context in paired-associates learning. American Journal of Psychology, 1969, 82, 415-424.

Biederman, I. Perceiving real-world scenes. Science, 1972, $177,77-80$

Collyer, S. C., Jonides, J., \& Bevan, W. Images as memory aids: Is bizarreness helpful? American Journal of Psychology, 1972, 85, 31-38.

CROWDER, R. G. Principles of learning and memory. Hillsdale, N.J: Lawrence Erlbaum, 1976.

KosSLYN, S. M. Information representation in visual images. Cognitive Psychology, 1975, 7, 341-370.

PAN, S. The influence of context upon learning and recall. Journal of Experimental Psychology, 1926, 9, 468-491.

Postman, L., \& Phillips, L. W. The effects of variable context on the acquisition and retention of paired associates. American Journal of Psychology, 1964, 77, 64-74.

Wollen, K. A., Weber, A., \& LoWry, D. Bizarreness versus interaction of mental images as determinants of learning. Cognitive Psychology, 1972, 3, 518-523. 